\title{
Multicomplex wave functions for linear and nonlinear Schrödinger equations
}

\author{
Kyle A. Theaker and Robert A. Van Gorder
}

\begin{abstract}
We consider a multicomplex Schrödinger equation with general scalar potential, a generalization of both the standard Schrödinger equation and the bicomplex Schrödinger equation of Rochon and Tremblay, for wave functions mapping onto $\mathbb{C}_{k}$. We determine the equivalent real-valued system in recursive form, and derive the relevant continuity equations in order to demonstrate that conservation of probability (a hallmark of standard quantum mechanics) holds in the multicomplex generalization. From here, we obtain the real modulus and demonstrate the generalized multicomplex version of Born's formula for the probability densities. We then turn our attention to possible generalizations of the multicomplex Schrödinger equation, such as the case where the scalar potential is replaced with a multicomplex-valued potential, or the case where the potential involves the real modulus of the wave function, resulting in a multicomplex nonlinear Schrödinger equation. Finally, in order to demonstrate the solution methods for such equations, we obtain several particular solutions to the multicomplex Schrödinger equation. We interpret the generalized results in the context of the standard results from quantum mechanics.
\end{abstract}

Mathematics Subject Classification (2010). Primary 30G35; Secondary 35J10.

Keywords. Multicomplex number system, Multicomplex analysis, Schrödinger equation.

\section{Introduction}

In the present work, we shall be interested in extending certain aspects of the mathematical formulation of quantum mechanics to the multicomplex number system. To begin with, note that bicomplex numbers, just like the quaternions, are a generalization of complex numbers to four real dimensions. They were introduced by Segre [42], and were created in order to bypass some of the negative features of quaternions. In particular, bicomplex numbers and 
quaternions differ in two important ways:

(i) quaternions form a division algebra, while bicomplex numbers do not, and (ii) bicomplex numbers are commutative, whereas quaternions are not.

For these reasons, the bicomplex number system has been shown to be more attractive (compared to the quaternions) for certain applications such as quantum mechanics [22, 23, 39, 40]. Bicomplex extensions of known functions have been carried out for the polygamma function [11], the Riemann zeta function [37], and the Hurwitz zeta function [12, 16]. Although bicomlex numbers are isomorphic to $\mathbb{R}^{4}$, bicomplex manifolds can also be defined [3], and this permits one to study a wide range of geometric structures via the bicomplex number formalism.

The attractive properties of bicomplex numbers are preserved when we define multicomplex numbers as the unique higher dimensional analogues to bicomplex numbers [9, 35]. One can extend analytic functions [18] to multicomplex variables, although one may also attempt to extend more complicated functions, such as those which are meromorphic. Recent theorems along these lines are given in [45]. For instance, the bicomplex Riemann zeta function was recently extended to a multicomplex Riemann zeta function [36]. A zeta function for multicomplex Algebras was very recently studied in [41]. Multicomplex variables have also found use in very practical applications, such as calculation of high order derivatives in computer algebra systems [21].

In this paper we consider the linear Schrödinger spectral problem

$$
i_{1} \hbar \Psi_{t}=-\frac{\hbar^{2}}{2 m} \Psi_{x x}+V(x, t) \Psi
$$

where $\Psi(x, t)$ is assumed continuous in its derivatives. Here, $\Psi: \mathbb{R}^{2} \rightarrow \mathbb{C}_{k}$ is the multicomplex wave function, $\mathbb{C}_{k}$ is the $k$ th multicomplex space, and $V: \mathbb{R}^{2} \rightarrow \mathbb{R}$ is the scalar potential. A multicomplex wave function $\Psi$ is a solution to (1.1), and can be written in the form

$$
\Psi(x, t)=\exp \left(\rho(x, t)+\theta(x, t) i_{i}\right),
$$

where $\rho(x, t)$ and $\theta(x, t)$ may be defined recursively. As mentioned above, the bicomplex linear Schrödinger spectral problem was previously considered in [39], and we can view this study as an extension of those results to the general multicomplex number case. Therefore, this will serve as a baseline for results in multicomplex quantum mechanics.

We first review properties of the multicomplex space $\mathbb{C}_{k}$ and then define several useful functions, in Section 2. In Section 3 we give the multicomplex Schrödinger equation and derive the continuity equations related to it for all possible forms of multicomplex conjugations. Physically, these continuity equations imply that probability is conserved for multicomplex wave functions. In Section 4, we define the real moduli and prove some theorems on properties of the wave function solutions to (1.1). As a consequence of these results, we recover the well-known Born's formula relating the squared modulus of the wave function (under principal moduli) to the probability density 
of measuring a particle being detected at a given place. We then extend the discussion to linear Schrödinger equations with non-scalar potentials $V(x, t)$. In particular, complex potentials have been considered in the literature on PT (Parity-Time) Symmetry (see [1, 4, 14, 17], among others), and one may actually study multicomplex potentials in the context of the linear Schrödinger spectral problem. We do so in Section 5, and such equations can be viewed as generalizations of those considered when studying PT symmetry. This generalization was previously considered in the bicomplex case - see [2] - and the relevant harmonic oscillator was solved. In Section 5, we also consider another extension of the results, to the case where the potential depends on the wave function modulus. Examples of such equations would be the cubic nonlinear Schrödinger equation or Gross-Pitaevskii equation [13, 34], which are applied to the study of Bose-Einstein condensates [7], nonlinear optics $[8,33]$, and quantum field theory [44], to name a few areas. In Section 6, we discuss how one would solve these multicomplex Schrödinger equations under certain physically relevant assumptions. Finally, in Section 7, we give concluding remarks.

\section{Properties of the space $\mathbb{C}_{k}$}

We begin this section with definitions that will prove useful throughout the remainder of the paper.

Definition 2.1. We define the multicomplex space $\mathbb{C}_{k}$ recursively by putting $\mathbb{C}_{0}=\mathbb{R}$ and $\mathbb{C}_{1}=\mathbb{C}$, then defining

$$
\mathbb{C}_{k}:=\left\{z_{1}+z_{2} i_{k}: z_{1}, z_{2} \in \mathbb{C}_{k-1}, i_{k}^{2}=-1, i_{p} i_{q}=i_{q} i_{p}, \text { for } p, q \leq k\right\}
$$

for every $k>1$. We also define $i_{0}=1$ and $i_{1}=i$. We call each $i_{k}$ a principal multicomplex unit. Products of the form $i_{p_{1}} i_{p_{2}} \ldots i_{p_{n}}$, where $n \leq k$ and $1 \leq$ $p_{1}<p_{2}<\ldots<p_{n} \leq k$ are called composite multicomplex units. Note that the set of all units of $\mathbb{C}_{k}$ forms a basis for $\mathbb{C}_{k}$. We denote this basis by $\mathbb{I}_{k}$. We denote by $\mathbb{I}_{k}^{p}$ the set of all units containing a factor of $i_{p}$.

Definition 2.2. For $z_{1}, z_{2}, z_{3}, z_{4} \in \mathbb{C}_{k-1}$, we define addition by

$$
\left(z_{1}+z_{2} i_{k}\right)+\left(z_{3}+z_{4} i_{k}\right)=\left(z_{1}+z_{3}\right)+\left(z_{2}+z_{4}\right) i_{k}
$$

and multiplication by

$$
\left(z_{1}+z_{2} i_{k}\right)\left(z_{3}+z_{4} i_{k}\right)=\left(z_{1} z_{3}-z_{2} z_{4}\right)+\left(z_{1} z_{4}+z_{2} z_{3}\right) i_{k},
$$

in analogy to the arithmetic of complex numbers. By further defining

$$
w_{1}\left(w_{2}+w_{3}\right)=w_{1} w_{2}+w_{1} w_{3}
$$

for $w_{1}, w_{2}, w_{3} \in \mathbb{C}_{k}$, the space $\mathbb{C}_{k}$ becomes an algebra over itself. 


\subsection{Expressing multicomplex numbers}

It should be noted that a multicomplex number $w=z_{1}+z_{2} i_{k} \in \mathbb{C}_{k}$ can be expressed with respect to any unit, and there are many idempotent representations for multicomplex numbers [43, 45]. By definition, we have

$$
\begin{aligned}
z_{1}+z_{2} i_{k} & =z_{11}+z_{12} i_{k-1}+\left(z_{21}+z_{22} i_{k-1}\right) i_{k} \\
& =\left(z_{11}+z_{21} i_{k}\right)+\left(z_{12}+z_{22} i_{k}\right) i_{k-1} .
\end{aligned}
$$

Proceeding inductively, we have

$$
z_{1}+z_{2} i_{k}=z_{1}^{\prime}+z_{2}^{\prime} i_{p}
$$

where $1 \leq p \leq k$ and $z_{1}^{\prime}, z_{2}^{\prime} \in \mathbb{C}_{k} / \mathbb{C}\left(i_{p}\right)$. Similarly, we can write

$$
z_{1}+z_{2} i_{k}=z_{1}^{\prime}+z_{2}^{\prime} i_{p_{1}} \ldots i_{p_{n}}
$$

with $1 \leq n \leq k$ and $z_{1}^{\prime}, z_{2}^{\prime} \in \mathbb{C}_{k} / \mathbb{C}\left(i_{p_{1}} \ldots i_{p_{n}}\right)$.

\subsection{Multicomplex conjugates}

Take $n \leq k$ and let $w \in \mathbb{C}_{k}$ be given by $w=z_{1}+z_{2} i_{m}$. Let us also recall that by $\dagger$ we mean the complex conjugation. That is, for any $q=p_{r}+i p_{1} \in \mathbb{C}$ with $p_{r}, p_{i} \in \mathbb{R}, q^{\dagger}=p_{r}-i p_{i}$. We shall extend this conjugation to multicomplex numbers, as follows.

Definition 2.3. For $1 \leq p_{1}<p_{2}<\ldots<p_{n} \leq k$, we define the multicomplex conjugate $\dagger_{\mathbf{p}}:=\dagger_{p_{1}, p_{2}, \ldots, p_{n}}$ as follows:

$$
w^{\dagger_{\mathbf{p}}}= \begin{cases}z_{1}^{\dagger_{\mathbf{p}}}+z_{2}^{\dagger \mathbf{p}} i_{p}, & \text { if } p \notin\left\{p_{1}, \ldots, p_{n}\right\}, \\ z_{1}^{\dagger \mathbf{q}}-z_{2}^{\dagger} i_{p}, & \text { if } p \in\left\{p_{1}, \ldots, p_{n}\right\},\end{cases}
$$

where $\left\{q_{1}, \ldots, q_{n-1}\right\}=\left\{p_{1}, \ldots, p_{n}\right\} \backslash\{m\}$.

For ease of notation, we will sometimes write $\dagger_{\mathbf{p}}:=\dagger_{p_{1}, \ldots, p_{n}}$ and $\dagger_{1, \ldots, \ell, \mathbf{p}}:=$ $\dagger_{1, \ldots, \ell, p_{n-\ell}, \ldots, p_{n}}$.

\subsection{Real and imaginary parts of a multicomplex number}

Take $w \in \mathbb{C}_{k}$ to be given by $w=z_{1}+z_{2} i_{p}$.

Definition 2.4. The real part of $w$ with respect to the $p$ th principal unit $i_{p}$, $\operatorname{Re}_{p}: \mathbb{C}_{k} \rightarrow \mathbb{C}_{k} / \mathbb{C}\left(i_{p}\right)$, is defined by

$$
\operatorname{Re}_{p}(w)=\operatorname{Re}_{p}\left(z_{1}+z_{2} i_{p}\right)=z_{1} .
$$

Definition 2.5. The imaginary part of $w$ with respect to the $p$ th principal unit $i_{p}, \operatorname{Im}_{p}: \mathbb{C}_{k} \rightarrow \mathbb{C}_{k} / \mathbb{C}\left(i_{p}\right)$, is defined by

$$
\operatorname{Im}_{p}(w)=\operatorname{Im}_{p}\left(z_{1}+z_{2} i_{p}\right)=z_{2} .
$$

Definition 2.6. More generally, we can define the functions

$$
\begin{aligned}
& \mathcal{R}_{\mathbf{p}}(w)=\frac{1}{2}\left(w+w^{\dagger} \mathbf{p}\right), \\
& \mathcal{I}_{\mathbf{p}}(w)=\frac{1}{2}\left(w-w^{\dagger} \mathbf{p}\right) .
\end{aligned}
$$


When conjugation in (2.4) is principal (that is, with respect to all principal units), these reduce to

$$
\mathcal{R}_{p}(w)=\operatorname{Re}_{p}(w), \quad \mathcal{I}_{p}(w)=\operatorname{Im}_{p}(w) i_{p}
$$

\subsection{Multicomplex moduli}

Let $\mathbf{p}=p_{1}, p_{2}, \ldots, p_{n}$ and $\mathbf{p}^{\prime}=p_{1}, p_{2}, \ldots, p_{n-1}$, where $n \leq k$ and $1 \leq p_{1}<$ $p_{2}<\ldots<p_{n} \leq k$. Take $w \in \mathbb{C}_{k}$ and write $w=z_{1}+z_{2} i_{k}$ for $z_{1}, z_{2} \in \mathbb{C}_{k-1}$.

Definition 2.7. The multicomplex $\operatorname{moduli}|\cdot|_{\mathbf{p}}=|\cdot|_{p_{1}, p_{2}, \ldots, p_{n}}$ are defined by $|w|_{\mathbf{p}}^{2}:=w w^{\dagger} \mathbf{p}$.

This definition gives the explicit formula

$$
|w|_{\mathbf{p}}^{2}= \begin{cases}\left.\left|z_{1}\right|_{\mathbf{p}}^{2}-\left|z_{2}\right|_{\mathbf{p}}^{2}+2 \mathcal{R}_{\mathbf{p}}\left(z_{1} z_{2}^{\dagger}\right)^{\dagger}\right), & \text { if } p_{n} \neq k \\ \left|z_{1}\right|_{\mathbf{p}^{\prime}}^{2}+\left|z_{2}\right|_{\mathbf{p}^{\prime}}^{2}-2 \mathcal{I}_{\mathbf{p}^{\prime}}\left(z_{1} z_{2}^{\dagger} \mathbf{p}^{\prime}\right), & \text { if } p_{n}=k\end{cases}
$$

\section{The Multicomplex Schrödinger Equation}

The Bicomplex Schrödinger equation of Rochon [39, 40] can be generalized to multicomplex functions by (1.1), where $\Psi: \mathbb{R}^{2} \rightarrow \mathbb{C}_{k}$ is the multicomplex wave function and $V: \mathbb{R}^{2} \rightarrow \mathbb{R}$ is the scalar potential.

The wave function $\Psi=\Psi(x, t)$ can be expressed in hyper-polar coordinates by the formula

$$
\Psi(x, t)=\exp \left(\rho(x, t)+\theta(x, t) i_{1}\right),
$$

where $\rho$ and $\theta$ are functions $\mathbb{R}^{2} \rightarrow \mathbb{C}_{k} / \mathbb{C}\left(i_{1}\right)$. Recall that properties of the function exp : $\mathbb{C}_{k} \rightarrow \mathbb{C}_{k}$ were discussed in Reid and Van Gorder [36]. (In particular, see Theorems 2,3,4 in [36].) With $\Psi$ expressed under the representation (3.1), we calculate

$$
\begin{gathered}
\Psi_{t}=\rho_{t} \exp \left(\rho+\theta i_{1}\right)+\theta_{t} \exp \left(\rho+\theta i_{1}\right) i_{1}, \\
i_{1} \Psi_{t}=-\theta_{t} \exp \left(\rho+\theta i_{1}\right)+i_{1} \rho_{t} \exp \left(\rho+\theta i_{1}\right), \\
\Psi_{x}=\rho_{x} \exp \left(\rho+\theta i_{1}\right)+\theta_{x} \exp \left(\rho+\theta i_{1}\right) i_{1}, \\
\Psi_{x x}=\left(\theta_{x x}+2 \rho_{x} \theta_{x}\right) \exp \left(\rho+\theta i_{1}\right)+\left(\rho_{x x}+\rho_{x}^{2}-\theta_{x}^{2}\right) \exp \left(\rho+\theta i_{1}\right) i_{1}, \\
V \Psi=V \exp \left(\rho+\theta i_{1}\right) .
\end{gathered}
$$

Placing (3.2)-(3.6) into (1.1), we obtain (after brief algebraic manipulations)

$$
\begin{aligned}
\left(\hbar \theta_{t}\right. & \left.-\frac{\hbar^{2}}{2 m}\left(\rho_{x}^{2}-\theta_{x}^{2}+\rho_{x x}\right)+V\right) \exp \left(\rho+\theta i_{1}\right) \\
& +\left(\hbar \rho_{t}+\frac{\hbar^{2}}{2 m}\left(2 \rho_{x} \theta_{x}+\theta_{x x}\right)\right) \exp \left(\rho+\theta i_{1}\right) i_{1}=0 .
\end{aligned}
$$

Recalling that $\rho, \theta \in \mathbb{C}_{k} / \mathbb{C}\left(i_{1}\right)$, we can separate equation (3.7) in the unit $i_{1}$. Doing so, we obtain a decomposition of the multicomplex Schrödinger equation into two partial differential equations, to wit.,

$$
\hbar \theta_{t}-\frac{\hbar^{2}}{2 m}\left(\rho_{x}^{2}-\theta_{x}^{2}+\rho_{x x}\right)+V=0
$$




$$
\hbar \rho_{t}+\frac{\hbar^{2}}{2 m}\left(2 \rho_{x} \theta_{x}+\theta_{x x}\right)=0
$$

\subsection{The multicomplex continuity equations}

Here we derive recursive forms for the multicomplex continuity equations. We begin by writing the Schrödinger equation under each possible type of conjugation:

$$
\begin{gathered}
i_{1} \hbar \Psi_{t}+\frac{\hbar^{2}}{2 m} \Psi_{x x}-V \Psi=0, \\
-i_{1} \hbar \Psi_{t}^{\dagger_{1}}+\frac{\hbar^{2}}{2 m} \Psi_{x x}^{\dagger_{1}}-V \Psi^{\dagger_{1}}=0, \\
i_{1} \hbar \Psi_{t}^{\dagger_{\mathbf{p}}}+\frac{\hbar^{2}}{2 m} \Psi_{x x}^{\dagger_{\mathbf{p}}}-V \Psi^{\dagger_{\mathbf{p}}}=0, \\
-i_{1} \hbar \Psi_{t}^{\dagger_{1, \mathbf{q}}}+\frac{\hbar^{2}}{2 m} \Psi_{x x}^{\dagger_{1, \mathbf{q}}}-V \Psi^{\dagger_{1, \mathbf{q}}}=0
\end{gathered}
$$

where $p_{1}>1$ and $q_{1}>1$.

We shall now show that the wavefunction solutions to the multicomplex Schrödinger equation conserve probability. That is, under the various conjugations (3.10)-(3.13), probability is conserved. The general conservation equation will take the form

$$
\frac{\partial}{\partial t}\{\text { probability density }\}+\frac{\partial}{\partial x}\{\text { probability current }\}=0 .
$$

In the multicomplex framework of our paper, "probability density" shall refer to any multicomplex analogue of the complex modulus $\Psi \Psi^{\dagger}$. When such equations are satisfied for all conjugations, we have that the multicomplex wave function retains the physical meaning of the standard complex wave function from quantum mechanics. On the other hand, when the right hand side of equation (3.14) is non-zero, probability is not conserved.

Theorem 3.1. Given a real-valued potential function $V(x, t)$ and a multicomplex wave function solution $\Psi(x, t)$ to the linear Schrödinger equation (1.1), probability will always be conserved as in (3.14).

Proof. The proof shall have four parts, corresponding to each conjugation given in (3.10)-(3.13). The first continuity equation: Multiplying (3.10) by $\Psi^{\dagger}$ and (3.11) by $\Psi$, we have

$$
\begin{aligned}
& i_{1} \hbar \Psi_{t} \Psi^{\dagger_{1}}+\frac{\hbar^{2}}{2 m} \Psi_{x x} \Psi^{\dagger_{1}}-V \Psi \Psi^{\dagger_{1}}=0 \\
& -i_{1} \hbar \Psi_{t}^{\dagger_{1}} \Psi+\frac{\hbar^{2}}{2 m} \Psi_{x x}^{\dagger_{1}} \Psi-V \Psi \Psi^{\dagger_{1}}=0 .
\end{aligned}
$$

Subtracting (3.16) from (3.15), we have

$$
\hbar\left(\Psi_{t} \Psi^{\dagger_{1}}+\Psi_{t}^{\dagger_{1}} \Psi\right) i_{1}+\frac{\hbar^{2}}{2 m}\left(\Psi_{x x} \Psi^{\dagger_{1}}-\Psi_{x x} \Psi\right)=0
$$


or equivalently,

$$
\left(\Psi \Psi^{\dagger_{1}}\right)_{t}+\frac{\hbar}{2 m i_{1}}\left(\Psi_{x x} \Psi^{\dagger_{1}}-\Psi_{x x}^{\dagger_{1}} \Psi\right)=0 .
$$

Define $\mathbf{J}_{1}[\Psi]:=\frac{\hbar}{2 m i_{1}}\left(\Psi_{x} \Psi^{\dagger_{1}}-\Psi_{x}^{\dagger_{1}} \Psi\right)$. Then,

$$
\left(\Psi \Psi^{\dagger_{1}}\right)_{t}+\left(\mathbf{J}_{1}[\Psi]\right)_{x}=0 .
$$

Now, recalling the hyper-polar representation of $\Psi$ given in (3.1), we have

$$
\begin{gathered}
\Psi^{\dagger_{1}}=\exp \left(\rho-\theta i_{1}\right), \\
\Psi_{x}=\left(\rho_{x}+\theta_{x} i_{1}\right) \exp \left(\rho+\theta i_{1}\right), \\
\Psi_{x}^{\dagger_{1}}=\left(\rho_{x}-\theta_{x} i_{1}\right) \exp \left(\rho-\theta i_{1}\right) .
\end{gathered}
$$

So,

$$
\Psi^{\dagger_{1}} \Psi_{x}-\Psi \Psi_{x}^{\dagger_{1}}=2 \theta_{x} i_{1} \exp (2 \rho)
$$

and therefore

$$
\mathbf{J}_{1}[\Psi]=\frac{\hbar}{m} \theta_{x} \exp (2 \rho) .
$$

The second continuity equation: For the next equation, we first multiply (3.10) by $\Psi^{\dagger 1, \mathbf{q}}$ and (3.13) by $\Psi$ to obtain the following equations:

$$
\begin{aligned}
& i_{1} \hbar \Psi_{t} \Psi^{\dagger_{1, \mathbf{q}}}+\frac{\hbar^{2}}{2 m} \Psi_{x x} \Psi^{\dagger_{1, \mathbf{q}}}-V \Psi \Psi^{\dagger_{1, \mathbf{q}}}=0, \\
& -i_{1} \hbar \Psi \Psi_{t}^{\dagger_{1, \mathbf{q}}}+\frac{\hbar^{2}}{2 m} \Psi \Psi_{x x}^{\dagger_{1, \mathbf{q}}}-V \Psi \Psi^{\dagger_{1, \mathbf{q}}}=0 .
\end{aligned}
$$

Subtracting (3.24) from (3.23) gives us

$$
\hbar\left(\Psi_{t} \Psi^{\dagger_{1, \mathbf{q}}}+\Psi_{t}^{\dagger_{1, \mathbf{q}}} \Psi\right) i_{1}+\frac{\hbar}{2 m}\left(\Psi_{x x} \Psi^{\dagger_{1, \mathbf{q}}}-\Psi_{x x}^{\dagger_{1, \mathbf{q}}} \Psi\right)=0,
$$

or equivalently,

$$
\left(\Psi \Psi^{\dagger_{1, \mathbf{q}}}\right)_{t}+\frac{\hbar}{2 m i_{1}}\left(\Psi_{x x} \Psi^{\dagger_{1, \mathbf{q}}}-\Psi_{x x}^{\dagger_{1, \mathbf{q}}} \Psi\right)=0 .
$$

Now we define the operator

$$
\begin{aligned}
\mathbf{J}_{1, \mathbf{q}}[\Psi] & :=\frac{\hbar}{2 m i_{1}}\left(\Psi_{x} \Psi^{\dagger_{1, \mathbf{q}}}-\Psi_{x}^{\dagger_{1, \mathbf{q}}} \Psi\right) \\
& =\frac{\hbar}{2 m i_{1}}\left(\left(\rho_{x}-\rho_{x}^{\dagger \mathbf{q}}\right)+\left(\theta_{x}+\theta_{x}^{\dagger \mathbf{q}}\right) i_{1}\right) \exp \left(\left(\rho+\rho^{\dagger \mathbf{q}}\right)+\left(\theta-\theta^{\dagger} \mathbf{q}\right) i_{1}\right) \\
& =\frac{\hbar}{2 m i_{1}}\left(\mathcal{I}_{\mathbf{q}}(\rho)+\mathcal{R}_{\mathbf{q}}(\theta) i_{1}\right)_{x} \exp \left(2\left(\mathcal{R}_{\mathbf{q}}(\rho)+\mathcal{I}_{\mathbf{q}}(\theta) i_{1}\right)\right) .
\end{aligned}
$$

Then from (3.26), we have the second continuity equation:

$$
\left(\Psi \Psi^{\dagger_{1, \mathbf{q}}}\right)_{t}+\left(\mathbf{J}_{1, \mathbf{q}}[\Psi]\right)_{x}=0 .
$$

The third continuity equation: to find our next continuity equation, we begin by multiplying (3.12) by $\Psi^{\dagger_{1}}$ and (3.11) by $\Psi^{\dagger_{p}}$, as such:

$$
i_{1} \hbar \Psi_{t}^{\dagger_{p}} \Psi^{\dagger_{1}}+\frac{\hbar^{2}}{2 m} \Psi_{x x}^{\dagger_{p}} \Psi^{\dagger_{1}}-V \Psi^{\dagger_{p}} \Psi^{\dagger_{1}}=0
$$




$$
-i_{1} \hbar \Psi_{t}^{\dagger_{1}} \Psi^{\dagger_{p}}+\frac{\hbar^{2}}{2 m} \Psi_{x x}^{\dagger_{1}} \Psi^{\dagger_{p}}-V \Psi^{\dagger_{1}} \Psi^{\dagger_{p}}=0
$$

Subtracting (3.30) from (3.29), we have

$$
\hbar\left(\Psi_{t}^{\dagger_{\mathbf{p}}} \Psi^{\dagger_{1}}+\Psi_{t}^{\dagger_{1}} \Psi^{\dagger_{\mathbf{p}}}\right) i_{1}+\frac{\hbar^{2}}{2 m}\left(\Psi_{x x}^{\dagger_{\mathbf{p}}} \Psi^{\dagger_{1}}-\Psi_{x x}^{\dagger_{1}} \Psi^{\dagger_{\mathbf{p}}}\right)=0
$$

or equivalently,

$$
\left(\Psi^{\dagger_{1}} \Psi^{\dagger_{p}}\right)_{t}+\frac{\hbar}{2 m i_{1}}\left(\Psi_{x x}^{\dagger_{p}} \Psi^{\dagger_{1}}-\Psi_{x x}^{\dagger_{1}} \Psi^{\dagger_{p}}\right)=0 .
$$

Defining the operator

$$
\begin{aligned}
\mathbf{J}_{1}^{\mathbf{p}}[\Psi] & :=\frac{\hbar}{2 m i_{1}}\left(\Psi_{x}^{\dagger_{\mathbf{p}}} \Psi^{\dagger_{1}}-\Psi_{x}^{\dagger_{1}} \Psi^{\dagger_{\mathbf{p}}}\right) \\
& =\frac{\hbar}{2 m i_{1}}\left(\left(\rho_{x}-\rho_{x}^{\dagger_{\mathbf{p}}}\right)+\left(\theta_{x}+\theta_{x}^{\dagger_{\mathbf{p}}}\right) i_{1}\right) \exp \left(\left(\rho+\rho^{\dagger_{\mathbf{p}}}\right)-\left(\theta-\theta^{\dagger_{\mathbf{p}}}\right) i_{1}\right) \\
& =\frac{\hbar}{2 m i_{1}}\left(\mathcal{I}_{\mathbf{p}}(\rho)+\mathcal{R}_{\mathbf{p}}(\theta) i_{1}\right)_{x} \exp \left(2\left(\mathcal{R}_{\mathbf{p}}(\rho)-\mathcal{I}_{\mathbf{p}}(\theta) i_{1}\right)\right),
\end{aligned}
$$

equation (3.32) gives us our next continuity equation:

$$
\left(\Psi^{\dagger_{1}} \Psi^{\dagger_{p}}\right)_{t}+\left(\mathbf{J}_{1}^{\mathbf{p}}[\Psi]\right)_{x}=0
$$

The fourth continuity equation: we multiply (3.12) by $\Psi^{\dagger_{1, \mathbf{q}}}$ and (3.13) by $\Psi^{\dagger} \mathbf{p}$ to obtain the following respective equations:

$$
\begin{gathered}
i_{1} \hbar \Psi_{t}^{\dagger_{\mathbf{p}}} \Psi^{\dagger_{1, \mathbf{q}}}+\frac{\hbar^{2}}{2 m} \Psi_{x x}^{\dagger_{\mathbf{p}}} \Psi^{\dagger_{1, \mathbf{q}}}-V \Psi^{\dagger_{\mathbf{p}}} \Psi^{\dagger_{1, \mathbf{q}}}=0, \\
-i_{1} \hbar \Psi_{t}^{\dagger_{1, \mathbf{q}}} \Psi^{\dagger_{\mathbf{p}}}+\frac{\hbar^{2}}{2 m} \Psi_{x x}^{\dagger_{1, \mathbf{q}}} \Psi^{\dagger_{\mathbf{p}}}-V \Psi^{\dagger_{1, \mathbf{q}}} \Psi^{\dagger_{\mathbf{p}}}=0 .
\end{gathered}
$$

Subtracting (3.36) from (3.35), we have

$$
\hbar\left(\Psi_{t}^{\dagger_{\mathbf{p}}} \Psi^{\dagger_{1, \mathbf{q}}}+\Psi_{t}^{\dagger_{1, \mathbf{q}}} \Psi^{\dagger_{\mathbf{p}}}\right) i_{1}+\frac{\hbar^{2}}{2 m}\left(\Psi_{x x}^{\dagger_{\mathbf{p}}} \Psi^{\dagger_{1, \mathbf{q}}}-\Psi_{x x}^{\dagger_{1, \mathbf{q}}} \Psi^{\dagger_{\mathbf{p}}}\right)
$$

or equivalently

$$
\left(\Psi^{\dagger} \mathbf{p} \Psi^{\dagger_{1, \mathbf{q}}}\right)_{t}+\frac{\hbar}{2 m i_{1}}\left(\Psi_{x x}^{\dagger_{\mathbf{p}}} \Psi^{\dagger_{1, \mathbf{q}}}-\Psi_{x x}^{\dagger_{1, \mathbf{q}}} \Psi^{\dagger_{\mathbf{p}}}\right) .
$$

Define

$$
\begin{aligned}
\mathbf{J}_{1, \mathbf{q}}^{\mathbf{p}}[\Psi]:= & \frac{\hbar}{2 m i_{1}}\left(\Psi_{x}^{\dagger_{\mathbf{p}}} \Psi^{\dagger_{1, \mathbf{q}}}-\Psi_{x}^{\dagger_{1, \mathbf{q}}} \Psi^{\dagger_{\mathbf{p}}}\right) \\
= & \frac{\hbar}{2 m i_{1}}\left(\left(\rho_{x}^{\dagger_{\mathbf{p}}}-\rho_{x}^{\dagger_{\mathbf{q}}}\right)+\left(\theta_{x}^{\dagger_{\mathbf{p}}}+\theta_{x}^{\dagger_{\mathbf{q}}}\right) i_{1}\right) \\
\quad & \times \exp \left(\left(\rho^{\dagger_{\mathbf{p}}}+\rho^{\dagger_{\mathbf{q}}}\right)+\left(\theta^{\dagger_{\mathbf{p}}}-\theta^{\dagger_{\mathbf{q}}}\right) i_{1}\right) \\
= & \frac{\hbar}{m i_{1}}\left\{\mathcal{I}_{\mathbf{p}}(\rho)-\mathcal{I}_{\mathbf{q}}(\rho)+\left(\mathcal{I}_{\mathbf{p}}(\theta)+\mathcal{R}_{\mathbf{q}}(\theta)\right) i_{1}\right\}_{x} \\
& \times \exp \left\{2\left(\mathcal{I}_{\mathbf{p}}(\rho)-\mathcal{R}_{\mathbf{q}}(\rho)+\left(\mathcal{I}_{\mathbf{p}}(\theta)+\mathcal{I}_{\mathbf{q}}(\theta)\right) i_{1}\right)\right\} .
\end{aligned}
$$


Then (3.38) becomes the fourth continuity equation:

$$
\left(\Psi^{\dagger_{p}} \Psi^{\dagger_{1, \mathbf{q}}}\right)_{t}+\left(\mathbf{J}_{1, \mathbf{q}}^{\mathbf{p}}[\Psi]\right)_{x}=0 .
$$

This concludes the proof.

It should be noted that this fourth continuity equation encompasses the other three equations. In particular, when $\mathbf{p}=\mathbf{q}$, we have

$$
\begin{aligned}
\mathbf{J}_{1, \mathbf{p}}^{\mathbf{p}}[\Psi] & =\frac{\hbar}{2 m i_{1}}\left(2 \theta_{x}^{\dagger_{\mathbf{p}}} i_{1}\right) \exp \left(2 \rho^{\dagger_{\mathbf{p}}}\right) \\
& =\frac{\hbar}{m} \theta_{x}^{\dagger_{\mathbf{p}}} \exp \left(2 \rho^{\dagger_{\mathbf{p}}}\right) .
\end{aligned}
$$

Thus, (3.40) reduces to $(3.19)$ with $\Psi^{\dagger}$ p replacing $\Psi$.

\section{Real moduli and the derivation of Born's formula}

In this section, we shall define the real moduli for the wave function, and then obtain some relevant results. One such result is that Born's formula holds under principal moduli for any complex wave function.

\subsection{Real moduli}

Take $w \in \mathbb{C}_{k}$ and define the family of operators $\Xi_{n}$ by

$$
\Xi_{n}[w]= \begin{cases}w, & \text { if } n=0 \\ \prod_{1 \leq p_{1}<\ldots<p_{n} \leq k} w^{\dagger_{p_{1}, \ldots, p_{n}},}, & \text { if } 1 \leq n \leq k .\end{cases}
$$

Note that

$$
\begin{gathered}
\Xi_{1}[w]=w^{\dagger_{1}} w^{\dagger_{2}} \ldots w^{\dagger_{k}} \\
\Xi_{2}[w]=w^{\dagger_{1,2}} w^{\dagger_{1,3}} \ldots w^{\dagger_{1, k}} w^{\dagger_{2,3}} w^{\dagger_{2,4}} \ldots w^{\dagger_{k-1, k}},
\end{gathered}
$$

and so on.

Definition 4.1. The real modulus $\|\cdot\|_{k}: \mathbb{C}_{k} \rightarrow \mathbb{R}$ is defined by

$$
\|w\|_{k}=\sqrt[2^{k}]{\prod_{m=0}^{k} \Xi_{m}[w]}
$$

Lemma 4.2. Consider a multicomplex-valued function of the form

$$
\exp \left(\sum_{e \in \mathbb{I}_{k}} \mu_{e} e\right)
$$

where $\mu_{e} \in \mathbb{C}_{k} / \mathbb{C}_{e}$ with $e \in \mathbb{I}_{k}$. Then

$$
\exp \left(\sum_{e \in \mathbb{I}_{k}} \mu_{e} e\right) \exp \left(\sum_{e \in \mathbb{I}_{k}} \mu_{e} e\right)^{\dagger_{p}}=\exp \left(2 \sum_{e \in \mathbb{I}_{k} \backslash \mathbb{I}_{k}^{p}} \mu_{e} e\right) \text {. }
$$


Proof. We have

$$
\begin{aligned}
\exp \left(\sum_{e \in \mathbb{I}_{k}} \mu_{e} e\right) \exp \left(\sum_{e \in \mathbb{I}_{k}} \mu_{e} e\right)^{\dagger_{p}}= & \exp \left(\sum_{e \in \mathbb{I}_{k} \backslash \mathbb{I}_{k}^{p}} \mu_{e} e+\sum_{e \in \mathbb{I}_{k}^{p}} \mu_{e} e\right) \\
& \times \exp \left(\sum_{e \in \mathbb{I}_{k} \backslash \mathbb{I}_{k}^{p}} \mu_{e} e+\sum_{e \in \mathbb{I}_{k}^{p}} \mu_{e} e\right)^{\dagger_{p}} \\
= & \exp \left(\sum_{e \in \mathbb{I}_{k} \backslash \mathbb{I}_{k}^{p}} \mu_{e} e+\sum_{e \in \mathbb{I}_{k}^{p}} \mu_{e} e\right) \\
& \times \exp \left(\sum_{e \in \mathbb{I}_{k} \backslash \mathbb{I}_{k}^{p}} \mu_{e} e-\sum_{e \in \mathbb{I}_{k}^{p}} \mu_{e} e\right) \\
= & \exp \left(2 \sum_{e \in \mathbb{I}_{k} \backslash \mathbb{I}_{k}^{p}} \mu_{e} e\right)
\end{aligned}
$$

which completes the proof.

Definition 4.3. For a multicomplex-valued function $\sum_{e \in \mathbb{I}_{k}} \mu_{e} e$, we define functions

$$
\begin{aligned}
& \nu_{0}=\mu_{1}, \\
& \nu_{1}=\mu_{i_{1}} i_{1}, \\
& \nu_{2}=\mu_{i_{2}} i_{2}+\mu_{i_{1} i_{2}} i_{1} i_{2},
\end{aligned}
$$

and in general for $n \leq k$, we define $\nu_{n}$ to be the sum of all terms from $\sum_{e \in \mathbb{I}_{k}} \mu_{e} e$ involving the unit $i_{n}$ but no terms involving units $i_{m}$ for $m<n$. Then

$$
\sum_{e \in \mathbb{I}_{k}} \mu_{e} e=\sum_{n=0}^{k} \nu_{n}
$$

Definition 4.4. Let $\bar{\nu}_{p}$ denote the partial sum

$$
\bar{\nu}_{p}=\sum_{n=0}^{p} \nu_{n}
$$

Theorem 4.5. For all $p=1, \ldots, k$, we have the reduction $\left\|\exp \left(\bar{\nu}_{p}\right)\right\|_{p}=$ $\left\|\exp \left(\bar{\nu}_{p-1}\right)\right\|_{p-1}$. 
Proof. Using Lemma 4.2, along with Definition 4.4 of $\bar{\nu}_{p}$ and Definition 4.1 of the real modulus $\|\cdot\|$, we have

$$
\begin{aligned}
& \left\|\exp \left(\bar{\nu}_{p}\right)\right\|_{p}=\sqrt[2^{p}]{\left|\prod_{j=0}^{p} \Xi_{j}\left[\exp \left(\bar{\nu}_{p}(x, t)\right)\right]\right|} \\
& =\sqrt[3]{\left|\left(\prod_{j=0}^{p-1} \Xi_{j}\left[\exp \left(\bar{\nu}_{p}\right)\right]\right)\left(\prod_{j=0}^{p-1} \Xi_{j}\left[\exp \left(\bar{\nu}_{p}\right)\right]\right)^{\dagger_{p}}\right|} \\
& =\sqrt[3]{\left|\left(\prod_{j=0}^{p-1} \Xi_{j}\left[\exp \left(\bar{\nu}_{p-1}\right)\right]\right)^{2}\right|} \\
& =\sqrt[2^{p-1}]{\left|\prod_{j=0}^{p-1} \Xi_{j}\left[\exp \left(\bar{\nu}_{p-1}\right)\right]\right|} \\
& =\left\|\exp \left(\bar{\nu}_{p-1}\right)\right\|_{p-1},
\end{aligned}
$$

which completes the proof.

Theorem 4.6. Fix $k \geq 1$ and let $\Psi$ be a multicomplex-valued function with the representation

$$
\Psi=\exp \left(\sum_{e \in \mathbb{I}_{k}} \mu_{e} e\right) .
$$

Then $\|\Psi\|_{k}=\exp \left(\mu_{1}\right)$.

Proof. We proceed by induction on $k$. When $k=1$, we have

$$
\begin{aligned}
\|\Psi\|_{1} & =\sqrt{\Xi_{0}[\Psi] \Xi_{1}[\Psi]} \\
& =\sqrt{\Psi \Psi^{\dagger}} \\
& =\sqrt{\exp \left(\mu_{1}+\mu_{i_{1}} i_{1}\right) \exp \left(\mu_{1}+\mu_{i_{1}} i_{1}\right)^{\dagger_{1}}} \\
& =\sqrt{\exp \left(\mu_{1}+\mu_{i_{1}} i_{1}\right) \exp \left(\mu_{1}-\mu_{i_{1}} i_{1}\right)} \\
& =\sqrt{\exp \left(2 \mu_{1}\right)} \\
& =\exp \left(\mu_{1}\right) .
\end{aligned}
$$

Now suppose that $k>1$. Using Theorem 4.5 , we have

$$
\begin{aligned}
\|\Psi\|_{k} & =\left\|\exp \left(\bar{\nu}_{k}\right)\right\|_{k} \\
& =\left\|\exp \left(\bar{\nu}_{k-1}\right)\right\|_{k-1} \\
& =\exp \left(\mu_{1}\right),
\end{aligned}
$$

where the last equality follows from our induction hypothesis. Thus, $\|\Psi\|_{k}=$ $\exp \left(\mu_{1}\right)$ for any $k \geq 1$. 
Corollary 4.7. Let the wave function $\Psi$ be defined as in Theorem 4.6. Then, $\|\Psi\|_{k}^{2}=\exp \left(2 \mu_{1}\right)$. This is exactly the standard Born formula.

We have shown that Born's formula holds under principal moduli. For non-principal moduli (resulting from non-principal conjugation), this relation need not hold. Indeed, is was shown in the bicomplex case [39] that when using non-principal conjugation, one obtains a sort of hyperbolic perturbation to Born's formula, suggesting that principal conjugation is needed for physically relevant extensions to the multicomplex case.

\section{Extensions of the linear Schrödinger spectral problem}

Now that we have understood general properties obeyed by multicomplex wave function solutions to the linear Schrödinger spectral problem, we consider two extensions. First, we shall generalize the potential function $V(x, t)$ so that it, too, is multicomplex-valued. This is in analogue to the complex potentials one sometimes encounters when working with Schrödinger equations in applications such as PT-Symmetry $[1,4,14,17]$. The second generalization we consider will be to the case where $V$ depends also on the modulus of the wave function solutions. This is encountered when working with nonlinear Schrödinger equations, which are encountered when studying nonlinear waves, Bose-Einstein condensates, nonlinear optics, and so on.

\subsection{Multicomplex-valued potentials $V$}

Consider equation (1.1) with $V=\mathcal{V}: \mathbb{R}^{2} \rightarrow \mathbb{C}_{k}$, i.e. with $\mathcal{V}(x, t)$ a multicomplexvalued function. This is in analogue to complex-valued potentials considered in the literature.

We will not recreate our calculations, but we can note that $V(x, t)$ can also be written in multicomplex polar form, as was done with the wave function in (3.1). However, for our purposes, it will be simpler to directly write

$$
\mathcal{V}(x, t)=\mathcal{V}_{1}(x, t)+\mathcal{V}_{2}(x, t) i_{1}
$$

where $\mathcal{V}_{1}$ and $\mathcal{V}_{2}$ are functions $\mathbb{R}^{2} \rightarrow \mathbb{C}_{k} / \mathbb{C}\left(i_{1}\right)$. Taking (3.1) and (5.1), we then obtain a decomposition of the multicomplex Schrödinger equation (1.1) into two differential equations as:

$$
\begin{gathered}
\hbar \theta_{t}-\frac{\hbar^{2}}{2 m}\left(\rho_{x}^{2}-\theta_{x}^{2}+\rho_{x x}\right)+\mathcal{V}_{1}=0, \\
\hbar \rho_{t}+\frac{\hbar^{2}}{2 m}\left(2 \rho_{x} \theta_{x}+\theta_{x x}\right)-\mathcal{V}_{2}=0
\end{gathered}
$$

Therefore, the potential will now directly enter into the time-evolution equation for the amplitude term in the $i_{1}$ unit, in addition to the equation for the time-evolution of the phase term in the $i_{1}$ unit. While this seems rather innocuous, as it suggests that the solution method will be only slightly more involved, let us now consider the continuity equations. As discussed in Section 3 , it suffices to consider the single continuity equation (3.41). If we recreate the computations used in the proof of Theorem 3.1 (multiplying the relevant 
modification of (3.12) by $\Psi^{\dagger_{1, \mathbf{q}}}$ and the relevant modification of (3.13) by $\left.\Psi^{\dagger} \mathbf{p}\right)$, we find that (3.40) is replaced with the continuity equation

$$
\left(\Psi^{\dagger_{p}} \Psi^{\dagger_{1, \mathbf{q}}}\right)_{t}+\left(\mathbf{J}_{1, \mathbf{q}}^{\mathbf{p}}[\Psi]\right)_{x}=\left(\mathcal{V}^{\dagger_{\mathbf{p}}}-\mathcal{V}^{\dagger_{1, \mathbf{q}}}\right) \Psi^{\dagger_{\mathbf{p}}} \Psi^{\dagger_{1, \mathbf{q}}} .
$$

As the quantity $\mathcal{V}^{\dagger} \mathbf{p}-\mathcal{V}^{\dagger 1, \mathbf{q}}$ is non-zero for arbitrary $\mathbf{p}$ and $\mathbf{q}$, the right hand side of equation (5.4) is non-zero. Hence, for multicomplex-valued potentials, probability is not, in general, conserved. This does not necessarily mean that the resulting equation is useless. Indeed, Schrödinger equations with generalized potentials are used in various areas, although for applications outside of quantum theory, and hence the lack of conserved probability is not a serious issue as the interpretation of equation (5.4) will be different.

Note that this framework might be useful for studying systems of equations, such as systems of linear Schrödinger equations with real-valued potentials, which are then transformed into a single multicomplex linear Schrödinger equation.

\subsection{Real-valued potentials $V$ which are dependent on the wave function modulus}

Let us now consider the case where $V$ is a real-valued function of $x, t$, as well as of the real modulus of the wave function, $\|\Psi\|_{k}$. (One can similarly define $V$ to depend on some other modulus, but this will perhaps be less physically relevant.) Then, we write $V=U\left(x, t,\|\Psi(x, t)\|_{k}\right): \mathbb{R}^{3} \rightarrow \mathbb{R}$, and we have the nonlinear Schrödinger equation

$$
i_{1} \hbar \Psi_{t}=-\frac{\hbar^{2}}{2 m} \Psi_{x x}+U\left(x, t,\|\Psi(x, t)\|_{k}\right) \Psi,
$$

In the case where $U\left(x, t,\|\Psi(x, t)\|_{k}\right)=\eta\|\Psi\|_{k}^{2}$ (where $\eta$ is a real-valued constant), (5.5) becomes the cubic nonlinear Schrödinger equation. When $U\left(x, t,\|\Psi(x, t)\|_{k}\right)=U_{0}(x, t)+\eta\|\Psi\|_{k}^{2}$, for a real-valued function $U_{0}$ of $x$ and $t,(5.5)$ becomes the Gross-Pitaevskii equation [13, 34]. These equations find application in areas such as Bose-Einstein condensation [7], nonlinear optics $[8,33]$, and quantum field theory [44].

Although the equation (5.5) is much more complicated to solve in general, since $U$ is real-valued and hence invariant under multicomplex conjugations, the results of Sections 3 and 4 remain valid.

Note that it is also possible to define $U$ as a multicomplex function, so that (5.5) is a nonlinear Schrödinger equation with multicomplex potential. However, this case will still present the physical difficulties (lack of conservation) in addition to the complications inherent in the solution method due to the nonlinearity.

\section{Stationary solutions for the multicomplex wave functions}

In the present section, we obtain several particular solutions, in order to compare and contrast the solution procedure for the multicomplex wave functions 
with those of the complex-valued wave functions from standard quantum theory. We focus on stationary solutions (solutions which are stationary under the real modulus), as the solution method is more tractible yet the solutions are still physically useful. We shall of course not be exhaustive, focusing on particular solutions that illustrate the solution procedure for the multicomplex case.

\subsection{Particular solutions for real-valued potential $V$}

We first consider stationary solutions in the case where the potential $V$ is real-valued. In the bicomplex case, the bicomplex harmonic oscillator was previously considered in an additional work $[2,10]$. Aside from this, we know of no other bicomplex or multicomplex wave function solutions to the linear Schrödinger equation.

Consider a stationary solution for the wave function solution to (1.1) which takes the form

$$
\Psi(x, t)=A(x) \exp \left(i_{1} \omega(t)\right),
$$

where $A, \omega \in \mathbb{C}_{k} / \mathbb{C}\left(i_{1}\right)$ are twice differentiable functions which must be determined. Let us assume an additively separable potential

$$
V(x, t)=V_{1}(x)+V_{2}(t),
$$

where both $V_{1}$ and $V_{2}$ are integrable functions of their respective arguments. This separable assumption still permits many of the interesting physical potentials. In fact, for most cases, one will have $V=V_{1}$, as potentials which vary in time are more rare. If we then separate variables through a spectral parameter $\lambda \in \mathbb{C}_{k} / \mathbb{C}\left(i_{1}\right)$ (in contrast to a real-valued spectral parameter used in the standard quantum theory), we obtain from (1.1) the system of ordinary differential equations

$$
\begin{gathered}
-\hbar \frac{d \omega}{d t}=V_{2}(t)+\lambda, \\
\frac{\hbar^{2}}{2 m} \frac{d^{2} A}{d x^{2}}+\left(\lambda-V_{1}(x)\right) A=0 .
\end{gathered}
$$

As $V_{2}$ is assumed integrable, the solution to equation (6.3) can always be written in the form

$$
\omega(t)=-\frac{1}{\hbar}\left(\lambda t+\int_{0}^{t} V_{2}(s) d s\right) .
$$

Meanwhile, the solution to equation (6.4) will depend strongly on the choice of $V_{1}$ and hence there is no general solution to this differential equation for arbitrary $V_{1}$. In the case where $V_{1}$ is periodic, we recover Hill's differential equation, for which there is no exact, closed-form solution [15]. Fourier series are often used to describe the solutions to such problems in terms of infinite series. The difficulty in solving such equations in general leads us to consider several examples. 
Example. Consider the constant potential in space, $V_{1}(x)=\hat{V}_{1}$, where $\hat{V}_{1}$ is a real-valued constant. Note that, as a special case when $\hat{V}_{1}=0$ and $V_{2}=0$, we recover the free particle potential [20]. The solution to (6.4) then reads

$$
A(x)=A_{0} \cos \left(\frac{\sqrt{2 m\left(\lambda-\hat{V}_{1}\right)}}{\hbar} x+\phi_{0}\right) .
$$

Here, cos is the extension of the cosine function to the multicomplex space $\mathbb{C}_{k}$ (as defined in Definition 1 of [36]), while $A_{0}, \phi_{0} \in \mathbb{C}_{k} / \mathbb{C}\left(i_{1}\right)$ are multicomplex constants. Therefore, when we take the scalar potential $V$ to have the form $V(x, t)=\hat{V}_{1}+V_{2}(t)$, we obtain particular wave function solutions to (1.1) of the form

$$
\Psi(x, t)=A_{0} \cos \left(\frac{\sqrt{2 m\left(\lambda-\hat{V}_{1}\right)}}{\hbar} x+\phi_{0}\right) \exp \left(-\frac{i_{1}}{\hbar}\left(\lambda t+\int_{0}^{t} V_{2}(s) d s\right)\right)
$$

which is multicomplex valued due to our taking the constants $\lambda, A_{0}, \phi_{0} \in$ $\mathbb{C}_{k} / \mathbb{C}\left(i_{1}\right)$. Otherwise, this solution is qualitatively the same as would have been found in the standard case for a complex-valued wave function.

Example. Consider the inverse square potential in space, $V_{1}(x)=\frac{\hat{V}_{1}}{x^{2}}$ (see [5] for a discussion on inverse square law potentials and their regularization), where $\hat{V}_{1}$ is a real-valued constant and $x>0$. The (bounded) solution to (6.4) then reads

$$
A(x)=A_{0} \sqrt{x} J_{\kappa}\left(\frac{\sqrt{2 m \lambda}}{\hbar} x\right),
$$

where $J_{\kappa}$ is the extension of the Bessel function of the first kind to the multicomplex space $\mathbb{C}_{k}$ (which is well-defined, since $J_{\kappa}$ is analytic), the index $\kappa$ is given by $\kappa=\frac{1}{2 \hbar} \sqrt{8 m \hat{V}_{1}+\hbar^{2}}$, and $A_{0} \in \mathbb{C}_{k} / \mathbb{C}\left(i_{1}\right)$ is a multicomplex constant. Therefore, when we take the scalar potential $V$ to have the form $V(x, t)=\frac{\hat{V}_{1}}{x^{2}}+V_{2}(t)$, we obtain particular wave function solutions to (1.1) of the form

$$
\Psi(x, t)=A_{0} \sqrt{x} J_{\kappa}\left(\frac{\sqrt{2 m \lambda}}{\hbar} x\right) \exp \left(-\frac{i_{1}}{\hbar}\left(\lambda t+\int_{0}^{t} V_{2}(s) d s\right)\right)
$$

for $x>0$, which is multicomplex valued due to our taking the constants $\lambda, A_{0} \in \mathbb{C}_{k} / \mathbb{C}\left(i_{1}\right)$. Again, this solution is qualitatively the same as would have been found in the standard case for a complex-valued wave function.

Example. Consider the triangular potential in space, $V_{1}(x)=\hat{V}_{1} x$ (see, for example, [25]), where $\hat{V}_{1}$ is a real-valued constant and $x>0$. The (bounded) solution to (6.4) then reads

$$
A(x)=A_{0} \operatorname{Ai}\left(\left(\frac{2 m}{\hbar^{2}}\right)^{1 / 3} \hat{V}_{1}^{-2 / 3}\left(\hat{V}_{1} x-\lambda\right)\right)
$$


where $\mathrm{Ai}$ is the extension of the Airy Ai function to the multicomplex space $\mathbb{C}_{k}$ (which is well-defined, since $\mathrm{Ai}$ is analytic) and $A_{0} \in \mathbb{C}_{k} / \mathbb{C}\left(i_{1}\right)$ is a multicomplex constant. Therefore, when we take the scalar potential $V$ to have the form $V(x, t)=\hat{V}_{1} x+V_{2}(t)$, we obtain particular wave function solutions to $(1.1)$ of the form

$$
\Psi(x, t)=A_{0} \operatorname{Ai}\left(\left(\frac{2 m}{\hbar^{2}}\right)^{1 / 3} \hat{V}_{1}^{-2 / 3}\left(\hat{V}_{1} x-\lambda\right)\right) \exp \left(-\frac{i_{1}}{\hbar}\left(\lambda t+\int_{0}^{t} V_{2}(s) d s\right)\right)
$$

for $x>0$, which is multicomplex valued due to our taking the constants $\lambda, A_{0} \in \mathbb{C}_{k} / \mathbb{C}\left(i_{1}\right)$. Again, this solution is qualitatively the same as would have been found in the standard case for a complex-valued wave function. Note that the Airy functions serve as a WKB approximation in the multicomplex context.

Example. Consider the Morse potential in space, $V_{1}(x)=\hat{V}_{1}(1-\exp (-x))^{2}$, where $\hat{V}_{1}$ is a real-valued constant (see [32]). One linearly independent solution to (6.4) then reads

$$
\begin{aligned}
& A(x)=A_{0} x^{1 / 2+\sqrt{2 m\left(\hat{V}_{1}-\lambda\right)} / \hbar} \\
& \quad \times \mathrm{M}\left(\frac{\sqrt{2 m\left(\hat{V}_{1}-\lambda\right)}-\sqrt{2 m \hat{V}_{1}}}{\hbar}+\frac{1}{2}, 1+2 \frac{\sqrt{2 m \hat{V}_{1}}}{\hbar} ; 2 \frac{\sqrt{2 m \hat{V}_{1}}}{\hbar} \exp (-x)\right)
\end{aligned}
$$

for $x>-1$, where $\mathrm{M}$ is the extension of Kummer's confluent hypergeometric function $\mathrm{M}$ to the multicomplex space $\mathbb{C}_{k}$ (which is well-defined for parameters where $\mathrm{M}$ is analytic) and $A_{0} \in \mathbb{C}_{k} / \mathbb{C}\left(i_{1}\right)$ is a multicomplex constant. The exponentiation of the factor $x$ is well-defined for any multicomplex $\lambda$, which follows from the results in [36]. Therefore, when we take the scalar potential $V$ to have the form $V(x, t)=\hat{V}_{1}(1-\exp (-x))^{2}+V_{2}(t)$, we obtain particular wave function solutions to (1.1) of the form

$$
\begin{aligned}
& \Psi(x, t)=A_{0} x^{1 / 2+\sqrt{2 m\left(\hat{V}_{1}-\lambda\right)} / \hbar} \\
& \quad \times \mathrm{M}\left(\frac{\sqrt{2 m\left(\hat{V}_{1}-\lambda\right)}-\sqrt{2 m \hat{V}_{1}}}{\hbar}+\frac{1}{2}, 1+2 \frac{\sqrt{2 m \hat{V}_{1}}}{\hbar} ; 2 \frac{\sqrt{2 m \hat{V}_{1}}}{\hbar} \exp (-x)\right) \\
& \quad \times \exp \left(-\frac{i_{1}}{\hbar}\left(\lambda t+\int_{0}^{t} V_{2}(s) d s\right)\right)
\end{aligned}
$$

for $x>-1$, which is multicomplex valued due to our taking the constants $\lambda, A_{0} \in \mathbb{C}_{k} / \mathbb{C}\left(i_{1}\right)$. This solution is qualitatively the same as would have been found in the standard case for a complex-valued wave function, although in this case we must be cautious of the fact that $M$ need not be analytic for all 
parameters. At such parameter values, the extension of $\mathrm{M}$ to the multicomplex numbers is then a more delicate matter, since it involves the extension of meromorphic, rather than analytic, functions to the multicomplex numbers.

These particular solutions demonstrate that stationary solutions to the multicomplex linear Schrödinger equation (1.1) keep the qualitative properties of their complex linear Schrödinger equation counterparts from quantum mechanics. In many cases, the solutions should simply be complexified versions of the standard solutions from quantum mechanics.

\subsection{Particular solutions for multicomplex-valued $V$}

Let us now consider the case where the potential is multicomplex valued, as in (5.1). Additionally, let us assume that the potential is additive separable, so that

$$
V(x, t)=\mathcal{V}(x, t)=\mathcal{V}_{11}(x)+\mathcal{V}_{12}(t)+\left(\mathcal{V}_{21}(x)+\mathcal{V}_{22}(t)\right) i_{1}
$$

Using the formulation given in (5.2)-(5.3), we also assume that

$$
\theta(x, t)=\theta_{1}(x)+\theta_{2}(t)
$$

and

$$
\rho(x, t)=\rho_{1}(x)+\rho_{2}(t),
$$

where $\theta_{1}, \theta_{2}, \rho_{1}, \rho_{2} \in \mathbb{C}_{k} / \mathbb{C}\left(i_{1}\right)$ are sufficiently differentiable functions of their arguments. Placing these into the system (5.2)-(5.3), we have that the multicomplex Schrödinger equation with multicomplex-valued potential is equivalent to the system

$$
\begin{gathered}
\hbar \frac{d \theta_{2}}{d t}+\mathcal{V}_{12}(t)-\lambda_{1}=0 \\
-\frac{\hbar^{2}}{2 m}\left(\left(\frac{d \rho_{1}}{d x}\right)^{2}-\left(\frac{d \theta_{1}}{d x}\right)^{2}+\frac{d^{2} \rho_{1}}{d x^{2}}\right)+\mathcal{V}_{11}(x)+\lambda_{1}=0 \\
\frac{\hbar^{2}}{2 m}\left(2 \frac{d \rho_{1}}{d x} \frac{d \theta_{1}}{d x}+\frac{d^{2} \theta_{1}}{d x^{2}}\right)-\mathcal{V}_{21}(x)+\lambda_{2}=0 \\
\hbar \frac{d \rho_{2}}{d t}-\mathcal{V}_{22}(t)-\lambda_{2}=0
\end{gathered}
$$

where $\lambda_{1}, \lambda_{2} \in \mathbb{C}_{k} / \mathbb{C}\left(i_{1}\right)$ are the multicomplex separation parameters. Clearly, we should find

$$
\theta_{2}(t)=\frac{1}{\hbar}\left(\lambda_{1} t-\int_{0}^{t} \mathcal{V}_{12}(s) d s\right)
$$

and

$$
\rho_{2}(t)=\frac{1}{\hbar}\left(\lambda_{2} t+\int_{0}^{t} \mathcal{V}_{22}(s) d s\right)
$$


Solving the remaining two equations (6.18)-(6.19) will be more involved. We can solve $(6.19)$ for $\theta_{1}(x)$ in terms of $\rho_{1}(x)$, obtaining

$$
\begin{aligned}
\theta_{1}(x)=K_{0} & +K_{1} \int_{0}^{x} \exp \left(-2 \rho_{1}(y)\right) d y \\
& +\frac{2 m}{\hbar^{2}} \int_{0}^{x} \int_{0}^{y}\left(\mathcal{V}_{21}(z)-\lambda_{2}\right) \exp \left(2\left(\rho_{1}(z)-\rho_{1}(y)\right)\right) d z d y .
\end{aligned}
$$

Here $K_{0}, K_{1} \in \mathbb{C}_{k} / \mathbb{C}\left(i_{1}\right)$ are constants. For simplicity, take $K_{0}=0$.

Substituting (6.23) into the remaining equation (6.18), we finally have an equation for only one unknown function:

$$
\begin{aligned}
\frac{d^{2} \rho_{1}}{d x^{2}} & +\left(\frac{d \rho_{1}}{d x}\right)^{2}-K_{1}^{2} \exp \left(-4 \rho_{1}\right) \\
& -\frac{4 m}{\hbar^{2}} K_{1} \exp \left(-2 \rho_{1}\right) \int_{0}^{x}\left(\mathcal{V}_{21}(y)-\lambda_{2}\right) \exp \left(2 \rho_{1}(y)\right) d y \\
& -\frac{4 m^{2}}{\hbar^{4}}\left(\int_{0}^{x}\left(\mathcal{V}_{21}(y)-\lambda_{2}\right) \exp \left(2 \rho_{1}(y)\right) d y\right)^{2} \\
& =\frac{2 m}{\hbar^{2}}\left(\lambda_{1}+\mathcal{V}_{11}(x)\right) .
\end{aligned}
$$

Equation (6.24) is a nonlinear integro-differential equation, which is nonautonomous. Therefore, it is impossible to solve in closed-form in all but the simplest special cases. Therefore, it must be solved numerically. Still, it provides us with an idea of how much more involved it is to solve the linear Schrödinger equation with multicomplex-valued potential.

\subsection{Particular solutions for nonlinear equations}

We shall not give a very detailed overview of solutions to the multicomplex nonlinear Schrödinger equation, as even in the standard case there has been innumerable work in this area (see, for instance, the recent papers $[29,30]$, and the many references therein). However, we will give one solution, to demonstrate that we recover the famous bright and dark soliton solutions for the one-dimensional spatial domain. For a more thorough summary of solutions to one-dimensional cubic nonlinear Schrödinger equations, see references [27, 28].

We consider equation (5.5) with the real-valued potential $U\left(x, t,\|\Psi(x, t)\|_{k}\right)=$ $\eta\|\Psi\|_{k}^{2}$. That is,

$$
i_{1} \hbar \Psi_{t}=-\frac{\hbar^{2}}{2 m} \Psi_{x x}+\eta\|\Psi\|_{k}^{2} \Psi,
$$

Here, $\eta \neq 0$ is a real-valued parameter. When $\eta>0,(5.5)$ is repulsive (see [27] and references therein) while when $\eta<0,(5.5)$ is attractive (see [28] and references therein). It makes sense to consider a stationary solution of the form

$$
\Psi(x, t)=R_{0} R(x) \exp \left(i_{1} \lambda t\right)
$$


where $\lambda \in \mathbb{C}_{k} / \mathbb{C}\left(i_{1}\right)$ is a multicomplex spectral parameter, $R_{0} \in \mathbb{C}_{k} / \mathbb{C}\left(i_{1}\right)$ is a multicomplex parameter, and $R \in \mathbb{C}_{k} / \mathbb{C}\left(i_{1}\right)$ is a multicomplex function of space alone.

For the repulsive regime, the standard cubic nonlinear Schrödinger equation have a solution of the form

$$
R(x)=\tanh \left(\frac{x}{\xi}\right),
$$

where $\xi$ is a parameter. This corresponds to a dark soliton. If we make such an assumption, we find that (6.25) gives the two algebraic conditions

$$
\eta\left\|R_{0}\right\|_{k}^{2} \xi^{2}=\frac{\hbar^{2}}{m} \quad \text { and } \quad \lambda \xi^{2}=\frac{\hbar}{m} .
$$

These conditions are interesting, in that they imply that $\lambda$ must be realvalued in order for such a solution to exist. Therefore, only the basis element along the $i_{1}$ coordinate can enter into the complex exponential to act as a phase; the remaining terms must all enter the amplitude term. Solving for $\lambda$ and $\xi$, we find that the dark soliton for the multicomplex cubic nonlinear Schrödinger equation is given by

$$
\Psi(x, t)=R_{0} \tanh \left(\frac{\left\|R_{0}\right\|_{k} \sqrt{\eta m}}{\hbar} x\right) \exp \left(i_{1} \frac{\eta\left\|R_{0}\right\|_{k}^{2}}{\hbar} t\right) .
$$

From this solution representation, we see that the multicomplex amplitude is a free parameter, and will strongly influence the shape of this dark soliton. Note that if other moduli were used in (6.25), then the solution structure would be more complicated. However, the real modulus is the closes analogue to what is standard in the study of, say, Bose-Einstein condensates.

For the attractive regime, we assume that

$$
R(x)=\operatorname{sech}\left(\frac{x}{\xi}\right),
$$

where again $\xi$ is a parameter. This assumption corresponds to a bright soliton. From (6.25), we obtain the two algebraic conditions

$$
|\eta|\left\|R_{0}\right\|_{k}^{2} \xi^{2}=\frac{\hbar^{2}}{m} \quad \text { and } \quad \lambda \xi^{2}=\frac{\hbar}{2 m} .
$$

Solving for $\lambda$ and $\xi$, we find that the bright soliton for the multicomplex cubic nonlinear Schrödinger equation is again determined by the free multicomplex parameter $R_{0}$, and is given by

$$
\Psi(x, t)=R_{0} \operatorname{sech}\left(\frac{\left\|R_{0}\right\|_{k} \sqrt{|\eta| m}}{\hbar} x\right) \exp \left(i_{1} \frac{|\eta|\left\|R_{0}\right\|_{k}^{2}}{\hbar} t\right) .
$$

While we have obtained dark and bright soliton solutions to the multicomplex analogue of the cubic nonlinear Schrödinger equation, of course other more complicated solutions are possible, and the solution of such equations would be a treatise in and of itself. Still, these results demonstrate that we are able to recover some very fundamental solutions arising in the study 
of Bose-Einstein condensates. This suggests that one might consider a theory of Bose-Einstein condensation in the multicomplex framework. Of course, a more involved study would be required in order to determine the utility and potential drawbacks of such an approach.

\section{Conclusions}

In summary, we have considered various attributes of a class of Schrödinger equations with multicomplex-valued wave function solutions. We derived the continuity equations for the multicomplex Schrödinger equation, which are then used to imply that probability is conserved for multicomplex wave functions, in analogy to the corresponding result from standard quantum mechanics. We then defined the real moduli, and were able to obtain the multicomplex analogue of Born's formula relating the squared modulus of the wave function to the probability density of measuring a particle being detected at a given place. We also considered two natural extensions of the multicomplex Schrödinger equations, namely linear Schrödinger equations with multicomplex-valued potentials $V(x, t)$ and then multicomplex nonlinear Schrödinger equations. We concluded our work by considering some stationary solutions to the multicomplex Schrödinger equations, in order to illustrate the similarities and differences between multicomplex wave function solutions and standard complex wave functions. For many cases, we found that the multicomplex wave functions retained many properties and qualitative features of the complex wave functions they generalize.

While the physical relevance of a multicomplex wave function may not be immediately clear, note that one can encode a vector of standard complex wave functions into a single multicomplex wave function. Then, instead of working with a system of complex Schrödinger equations, one can study a single multicomplex Schrödinger equation. In the linear case, we have seen that several particular solutions to the complex Schrödinger equation carry over the the multicomplex case. Therefore, when relevant, it may be more efficiency to solve a single multicomplex equation than a large number of complex equations.

We have considered the multicomplex wave function for the Schrödinger equation on the spatial domain $\mathbb{R}$, that is $\Psi(x, t): \mathbb{R}^{2} \rightarrow \mathbb{C}_{k}$. The extension of these results to the Schrödinger equation on the spatial domain $\mathbb{R}^{n}, n \geq 2$, that is $\Psi(x, t): \mathbb{R}^{n+1} \rightarrow \mathbb{C}_{k}$, is straightforward for the linear case, yet much more cumbersome in terms of notation. Note that one might also extend our results to Schrödinger equations defined over multicomplex domains, that is $\Psi(x, t): \mathbb{C}_{n} \times \mathbb{R} \rightarrow \mathbb{C}_{k}$ (for $n>1$ not necessarily equal to $k>1$ ). That would involve the study of the Laplacian on multicomplex numbers, and would continue work studying the behavior of Schrödinger equations on manifolds. Work along these lines has already been considered by Rochon [38], who extended the study of Schrödinger equations to the case where the wave functions were defined over bicomplex numbers. This would be one possible 
extension of the Schrödinger equation to manifold domains. For recent work along these lines, see $[6,47,48]$ and references therein. Furthermore, the Laplacian on bicomplex numbers was recently studied in [26]. Also relevant to this goal, recent work on first-order differential equations defined over multicomplex spaces was given in [43].

In some of the particular solutions discussed in Section 6, we discovered that multicomplex spectral parameters were possible. There is a rich area of research on the relation between spectral parameter values and whether a wave is orbitally stable or unstable $[19,31,46,49]$. It would be interesting to consider what effect the multicomplex property of the spectral parameters might have on stability of waves, particularly the stability of nonlinear wave solutions to various kinds of multicomplex nonlinear Schrödinger equations of the form (5.5). This would be an interesting area for future work.

\section{References}

[1] Z. Ahmed, Real and complex discrete eigenvalues in an exactly solvable onedimensional complex PT-invariant potential, Physics Letters A 282 (2001) 343348.

[2] B. Bagchi and A. Banerjee, Bicomplex hamiltonian systems in quantum mechanics, Journal of Physics A: Mathematical and Theoretical 48 (2015) 505201.

[3] P. Baird and J. C. Wood, Harmonic Morphisms and Bicomplex Manifolds, Journal of Geometry and Physics 61 (2011) 46-61.

[4] C. M. Bender and S. Boettcher, Quasi-exactly solvable quartic potential, Journal of Physics A: Mathematical and General 31(14) (1998) L273.

[5] D. Bouaziz and M. Bawin, Regularization of the singular inverse square potential in quantum mechanics with a minimal length, Physical Review A 76 (2007) 032112 .

[6] M. Bureš, Energy spectrum of the hydrogen atom in a space with one compactified extra dimension, $R^{3} \times S^{1}$, Annals of Physics 363 (2015) 354-363.

[7] F. Dalfovo, S. Giorgini, L.P. Pitaevskii, and S. Stringari, Theory of Bose-Einstein condensation in trapped gases, Reviews of Modern Physics 71(3) (1999) 463.

[8] S. Dyachenko, A. C. Newell, A. Pushkarev, and V. E. Zakharov, Optical turbulence: weak turbulence, condensates and collapsing filaments in the nonlinear Schrödinger equation, Physica D 57 (1992) 96-160.

[9] V. Garant-Pelletier and D. Rochon, On a Generalized Fatou-Julia Theorem in Multicomplex Spaces, Fractals 17 (2009) 241-255.

[10] R. Gervais Lavoie, L. Marchildon, and D. Rochon, The bicomplex quantum harmonic oscillator, Il Nuovo Cimento B 125 (2010) 1173-1192.

[11] R. Goyal, Bicomplex Polygamma Function, Tokyo Journal of Mathematics 30 (2007) 523-530.

[12] S.P. Goyal and R. Goyal, On Bicomplex Hurwitz Zeta Function, South East Asian Journal of Mathematics and Mathematical Sciences 4 (2006) 59-66.

[13] E. P. Gross, Structure of a quantized vortex in boson systems, Il Nuovo Cimento 20(3) (1961) 454-457. 
[14] A. Guo, G. J. Salamo, D. Duchesne, R. Morandotti, M. Volatier-Ravat, V. Aimez, G. A. Siviloglou, and D. N. Christodoulides, Observation of P Tsymmetry breaking in complex optical potentials, Physical Review Letters 103 (2009) 093902.

[15] G.W. Hill, On the Part of the Motion of Lunar Perigee Which is a Function of the Mean Motions of the Sun and Moon, Acta Mathematica 8(1) (1886) 1-36.

[16] A. Javtokas, A bicomplex Hurwitz zeta-function, S'iauliai Mathematical Seminar 1(9) (2006) 23-31.

[17] C.-S. Jia, X.-L. Zeng, and L.-T. Sun, PT symmetry and shape invariance for a potential well with a barrier, Physics Letters A 294 (2002) 185-189.

[18] P.W. Ketchum, Analytic functions of hypercomplex variables, Trans. Amer. Math. Soc 30 (1928) 641-667.

[19] E.A. Kuznetsov, A.M. Rubenchik, and V.E. Zakharov, Soliton stability in plasmas and hydrodynamics, Physics Reports 142(3) (1986) 103-165.

[20] L.D. Landau and E.M. Lifshits, Quantum Mechanics, Non-relaticistic Theory. Perganon Press, 1965.

[21] G. Lantoine, R.P. Russel and T. Dargent, Using Multicomplex Variables for Automatic Computation of High-Order Derivatives, ACM Transactions on Mathematical Software 38 (2012) Article No. 16 (doi: 10.1145/2168773.2168774).

[22] R.G. Lavoie, L. Marchildon and D. Rochon, The bicomplex quantum harmonic oscillator, Il Nuovo Cimento 125 B (2010) 1173-1192.

[23] R.G. Lavoie, L. Marchildon and D. Rochon, Finite-Dimensional Bicomplex Hilbert Spaces, Advances in Applied Clifford Algebras 21 (2011) 561-581.

[24] J.-Q. Li, Y.-G. Miao, and Z. Xue, A possible method for non-Hermitian and non-PT-symmetric Hamiltonian systems, PLoS One 9(6) (2014) e97107.

[25] W.W. Lui and M. Fukuma, Exact solution of the Schrodinger equation across an arbitrary one-dimensional piecewise-linear potential barrier, Journal of Applied Physics 60 (1986) 1555-1559.

[26] M. E. Luna-Elizarraras, M. Shapiro, D. C. Struppa, and A. Vajiac, Complex Laplacian and derivatives of bicomplex function, Complex Analysis and Operator Theory 7(5) (1013) 1675-1711.

[27] K. Mallory and R.A. Van Gorder, Stationary solutions for the $1+1$ nonlinear Schrödinger equation modeling repulsive Bose-Einstein condensates in small potentials, Physical Review E 88 (2013) 013205.

[28] K. Mallory and R.A. Van Gorder, Stationary solutions for the $1+1$ nonlinear Schrödinger equation modeling attractive Bose-Einstein condensates in small potentials, Physical Review E 89 (2014) 013204.

[29] K. Mallory and R.A. Van Gorder, Stationary solutions for the $2+1$ nonlinear Schrödinger equation modeling Bose-Einstein condensates in radial potentials, Physical Review E 90 (2014) 023201.

[30] K. Mallory and R.A. Van Gorder, Stationary solutions for the nonlinear Schrödinger equation modeling three-dimensional spherical Bose-Einstein condensates in general potentials, Physical Review E 92 (2015) 013201.

[31] B.A. Malomed, D.J. Kaup, and R.A. Van Gorder, Unstaggered-staggered solitons in two-component discrete nonlinear Schrödinger lattices, Physical Review E 85 (2012) 026604. 
[32] P.M. Morse, Diatomic molecules according to the wave mechanics. II. Vibrational levels, Physical Review 34 (1929) 57.

[33] A. Picozzi, J. Garnier, T. Hansson, P. Suret, S. Randoux, G. Millot, and D. N. Christodoulides, Optical wave turbulence: Towards a unified nonequilibrium thermodynamic formulation of statistical nonlinear optics, Physics Reports 542 (2014) 1-132.

[34] L.P. Pitaevskii, Vortex Lines in an Imperfect Bose Gas, Soviet Physics JETP 13(2) (1961) 451-454.

[35] G. B. Price, An Introduction to Multicomplex Spaces and Functions, Marcel Dekker (1991).

[36] F. L. Reid and R. A. Van Gorder, A multicomplex Riemann zeta function, Advances in Applied Clifford Algebras 23 (2013) 237-251.

[37] D. Rochon, A Bicomplex Riemann Zeta Function, Tokyo Journal of Mathematics 27 (2004) 357-369.

[38] D. Rochon, On a relation of bicomplex pseudoanalytic function theory to the complexified stationary Schrödinger equation, Complex Variables and Elliptic Equations 53 (2008) 501-521.

[39] D. Rochon and S. Tremblay, Bicomplex quantum mechanics: I. The generalized Schrödinger equation, Advances in Applied Clifford Algebras 14 (2004) 231-248.

[40] D. Rochon and S. Tremblay, Bicomplex quantum mechanics: II. The Hilbert space, Advances in Applied Clifford Algebras 16 (2006) 135-157.

[41] A. Sebbar, D. C. Struppa, A. Vajiac, and M. B. Vajiac, A Zeta Function for Multicomplex Algebra. Preprint (2016) arXiv:1601.04785.

[42] C. Segre, The real representation of complex elements and hyperalgebraic entities (Italian), Mathematische Annalen 40 (1892) 413-467.

[43] D. C. Struppa, A. Vajiac, and M. B. Vajiac, Differential Equations in Multicomplex Spaces, Hypercomplex Analysis: New Perspectives and Applications. Springer International Publishing, 2014. pp. 213-227.

[44] H.B. Thacker, Exact integrability in quantum field theory and statistical systems, Reviews of Modern Physics 53(2) (1981) 253.

[45] A. Vajiac and M.B. Vajiac, Multicomplex Hyperfunctions, Complex Variables and Elliptic Equations 57 (2012) 751-762.

[46] N.G. Vakhitov and A.A. Kolokolov, Stationary solutions of the wave equation in the medium with nonlinearity saturation, Radiophysics and Quantum Electronics 16 (1973) 783-789.

[47] R.A. Van Gorder, Wave functions and energy spectra for the hydrogenic atom in $\mathbb{R}^{3} \times \mathcal{M}$, Journal of Mathematical Physics 51 (2010) 122104 .

[48] R.A. Van Gorder, Schrödinger equations on $\mathbb{R}^{3} \times \mathcal{M}$ with non-separable potential, Journal of Mathematical Chemistry 50 (2012) 1420-1436.

[49] R.A. Van Gorder, Orbital Instability of the Peregrine Soliton, Journal of the Physical Society of Japan 83 (2014) 054005. 
K. A. Theaker and R. A. Van Gorder

Kyle A. Theaker

Department of Mathematics

University of Central Florida

Orlando, FL 32816-1364 USA

e-mail: kyle.theaker@ucf .edu

Robert A. Van Gorder

Mathematical Institute

University of Oxford

Andrew Wiles Building

Radcliffe Observatory Quarter, Woodstock Road

Oxford, OX2 6GG, United Kingdom

e-mail: Robert.VanGorder@maths.ox.ac.uk 